\title{
Neural Network Blind Equalization Algorithm Applied in Medical CT Image Restoration
}

\author{
Yunshan Sun, ${ }^{1,2}$ Liyi Zhang, ${ }^{1,2}$ Jin Zhang, ${ }^{3}$ and Lijuan Shi ${ }^{1}$ \\ ${ }^{1}$ School of Information Engineering, Tianjin University of Commerce, Tianjin 300134, China \\ ${ }^{2}$ School of Electric Information Engineering, Tianjin University, Tianjin 300072, China \\ ${ }^{3}$ Shanxi Academy of Medical Sciences, Shanxi Dayi Hospital, Taiyuan 030024, China
}

Correspondence should be addressed to Liyi Zhang; zhangliyi@tjcu.edu.cn

Received 21 November 2012; Revised 21 January 2013; Accepted 8 February 2013

Academic Editor: Erwei Bai

Copyright (C) 2013 Yunshan Sun et al. This is an open access article distributed under the Creative Commons Attribution License, which permits unrestricted use, distribution, and reproduction in any medium, provided the original work is properly cited.

\begin{abstract}
A new algorithm for iterative blind image restoration is presented in this paper. The method extends blind equalization found in the signal case to the image. A neural network blind equalization algorithm is derived and used in conjunction with Zigzag coding to restore the original image. As a result, the effect of PSF can be removed by using the proposed algorithm, which contributes to eliminate intersymbol interference (ISI). In order to obtain the estimation of the original image, what is proposed in this method is to optimize constant modulus blind equalization cost function applied to grayscale CT image by using conjugate gradient method. Analysis of convergence performance of the algorithm verifies the feasibility of this method theoretically; meanwhile, simulation results and performance evaluations of recent image quality metrics are provided to assess the effectiveness of the proposed method.
\end{abstract}

\section{Introduction}

As one of the important methods in medical image diagnosis, CT image can achieve a high performance of visualizing and measuring microstructure of apparatus and tissue on detecting small lesions. However, CT has an issue that the recorded image represents a noisy and blurred version of the original scene, which is also common on many other applications such as astronomical imaging [1], sonar imaging [2], remote imaging [3], microscopy imaging [4], and medical ultrasound imaging $[5,6]$. On one hand, this issue is caused by one of the facts that CT scanners cannot resolve many important temporal details, where part of the images was degraded by the underlying point spread function. One of the effective strategies to tackle this issue is digital image restoration, which is aimed at image resolution enhancement. On the other hand, the underlying point spread function (PSF) of the CT scanner is often unavailable in practice. Therefore, we develop a deblurring approach for resolution improvement of CT image without prior knowledge, or only based on partial and poor knowledge of the characteristics of the original image and the noise. In this paper, we will address the only problem by utilizing the degraded image only in order to obtain an estimation of the original scene and present a novel blind image restoration algorithm.

Various methods have been proposed in the previous literatures to address the image restoration problem. These image restoration methods can be divided into two main classes [7]. The first class contains methods that separate blur identification as a disjoint procedure from restoration, such as maximum likelihood [8], generalized cross-validation [9], zero sheet separation [10], Bayesian estimation [11], maximum a posteriori [12], and expectation maximization [13]. The second class consists of methods that combine blur identification and restoration in one procedure, such as the iterative blind deconvolution techniques [14], the nonnegativity support constraints recursive inverse filtering $[15,16]$, simulated annealing [17], and total variation blind image deconvolution [18].

The neural network approach for image restoration in both types is a relatively active topic. Artificial neural network has been studied for many years in the hope of achieving human-like performance via dense interconnections of simple computational elements. Based on our present 
understanding of biological nervous systems, neural networks have their greatest potential in areas such as signal processing and image recognition, in which many hypotheses are pursued in parallel and high computation rates and adoption are required, but the current best systems are still far from equaling human performance. Cheema et al. [19] presented an image restoration algorithm to identify noncausal blur function. The noisy and blurred images are modeled as continuous associative networks, whereas autoassociative part determines the image model coefficients and the heteroassociative part determines the blur function of the system. The neural network model combining an adaptive auto-associative network with a random Gaussian process is proposed to restore the blurred image and blur function simultaneously. In the literature [20], a nonlinear restoration of diffraction-limited images concurrently with quantization was accomplished in the process of image compression utilizing vector quantization neural network. Here, neural network is trained on image pairs consisting of a lossless compression named hierarchical vector quantization. Igor Aizenberg et al. [21] proposed a multilayer neural network with a traditional feedforward architecture image restoration algorithm based on multivalued neurons. A new image-reconstruction algorithm using the cellular neural network was presented to solve the Navier-Stokes equation and offer a robust method for estimating the background signal within the gene-spot region in [22]. Wu et al. [23] proposed the modified Hopfield neural network model for grey image restoration, which avoids the shortcomings of Zhou's model, improves convergence speed, and possesses superior edge preserving performance.

Blind equalization algorithm is to estimate the unknown system parameters and structure based on the system output but without prior knowledge of the inputting signal and the blur. Zhang et al. [24] proposed several blind equalization algorithms based on neural network, which present better performances such as quick convergence speed and small steady error in eliminating intersignal interface. The process of medical CT image degradation on constructing CT diagnosis image in medical image applications is equivalent to the formation process of inter-signal interface in the digital communication. Therefore, we develop a blind neural network approach for blind restoration of medical CT images.

The neural network approach for blind image restoration proposed in this paper belongs to the second class. Preliminary results of blind equalization algorithm were first found in the signal case and then extended to the image. Secondly, a neural network blind equalization, which makes use of the similarity between the image degradation process and ISI, is derived and utilized in conjunction with Zigzag coding to restore the original image. We proposed the way of adopting Zigzag coding to transform the degradation image into a one-dimensional signal sequence. Thirdly, a one-dimensional signal sequence CMA cost function is also established and optimized by the gradient descent method to obtain the optimal estimation of the transformed signal. Meanwhile, a neural network blind equalization algorithm is adopted to eliminate the impact of point spread function. Compared to the existing algorithm, the novel method avoids twodimensional matrix inverse iteration operation, reduces the complexity of the algorithm, and effectively improves restoration as well. Eventually, simulation results verify the effectiveness of the algorithm.

The remainder of this paper is organized as follows. In Section 2, the image and degradation models are reviewed, the formation of Zigzag coding is introduced, and the structure of feedforward neural network is revisited. The neural network blind equalization algorithm is derived and used in conjunction with Zigzag coding to restore the original image. Alternating minimization of the cost function is reviewed in Section 3. The convergence performance of the proposed algorithm is analyzed in Section 4. Simulation results obtained with simulated noisy blurred images are given in Section 5, and concluding remarks are given in Section 6.

\section{Preliminary Knowledge}

2.1. Degradation Model. In most cases, a linear blur and an additive noise process can be used to model the degradation process, which is commonly given by

$$
\begin{aligned}
g(i, j) & =f(i, j) * h(i, j)+n(i, j) \\
& =\sum_{k=0}^{M} \sum_{l=0}^{N} f(k, l) h(i-k, j-l)+n(i, j),
\end{aligned}
$$

in which $g(i, j), f(i, j)$ and $n(i, j)$, are images represent the degraded image, the original image, and additive noise, respectively; $h(i, j)$ is the PSF of the blurring operator, and $*$ denotes the mathematical operation of convolution.

The purpose of blind image restoration is to estimate the original image without prior knowledge, or based on partial and poor knowledge of the characteristics of $f(i, j)$ and $h(i, j)$, to obtain the estimation $\widehat{f}(i, j)$ of the original.

2.2. Zigzag Coding. In order to transform image into a 1-dimensional signal sequence, zigzag coding process is described with each element of image vector scanned by a simple zigzag graph. A zigzag array is a square arrangement of the first $\mathrm{N}^{2}$ integers, where the numbers increase sequentially as you zigzag along the antidiagonals of the array. The scrambling of matrix element is implemented by the above-mentioned course, and this process is called zigzag coding. For a graphical representation, the general structure of a zigzag coding is depicted in Figure 1.

In order to utilize blind equalization technology to complete medical CT image blind restoration, firstly, two-dimensional medical degradation CT images should be transformed into one-dimensional signal sequence by zigzag coding discussed previously.

2.3. Feedforward Neural Network. There exist several different neural network architectures available for medical imaging applications, but the most common one of them is the feedforward neural network. In a feedforward neural network, the neurons in each layer are only connected with the neurons in the next layer. These connections are unidirectional, which means that signals or information being 


\begin{tabular}{|c|c|c|c|c|}
\hline 1 & 2 & 3 & 4 & 5 \\
\hline 6 & 7 & 8 & 9 & 10 \\
\hline 11 & 12 & 13 & 14 & 15 \\
\hline 16 & 17 & 18 & 19 & 20 \\
\hline 21 & 22 & 23 & 24 & 25 \\
\hline
\end{tabular}

(a) The original data matrix

\begin{tabular}{|l|l|l|l|l|l|l|l|l|l|l|l|l|l|l|l|l|l|l|}
\hline 1 & 6 & 2 & 3 & 7 & 11 & 16 & 12 & 8 & 4 & 5 & 9 & 13 & 17 & 21 & 22 & 18 & 14 & $\cdots$ \\
\hline
\end{tabular}

(b) The sequence after Zigzag coding

FIgURe 1: Zigzag coding.

processed can only pass through the network in a single direction, from the input layer through the hidden layer to the output layer. The most usual feedforward NN is composed of 3 layers: an input layer, a hidden layer, and an output layer. The structure of the described neural network is illustrated in Figure 2 [25].

The above-mentioned three-layer feedforward neural network structure can be addressed to eliminate the effect of PSF and obtain the estimation of the original image. $w_{l k}(n)$ denotes the weight between the input layer and the hidden layer, $l$ describes the index of the input neuron $(l=0,1$, $\ldots, L-1), w_{k}(n)$ denotes the weight between the hidden layer and the output layer, and $k$ describes the index of the hidden neuron $(k=0,1, \ldots, K)$.

Suppose that the input of the input layer is given by

$$
\mathbf{G}(n)=[g(n), g(n-1), \ldots, g(n-L+1)]^{T} .
$$

For the sake of notational simplicity, we denote the input of the hidden layer by $u_{k}(n)$, define $I_{k}(n)$ as the output of the hidden layer, describe $v(n)$ as the output of the output layer, and utilize $\tilde{f}(n)$ as the output of neural network. State equations of feedforward neural network are represented as

$$
\begin{gathered}
u_{k}(n)=\sum_{l=0}^{L-1} w_{l k}(n) g(n-l), \\
I_{k}(n)=q\left[u_{k}(n)\right], \\
v(n)=\sum_{k=0}^{K} w_{k}(n) I_{k}(n), \\
\tilde{f}(n)=q[v(n)],
\end{gathered}
$$

where $q(\cdot)$ denotes the transfer function not only between the hidden layer and the output layer, but also between the input layer and the hidden layer.

As seen, $L$ input units are included in the input layer, which is decided by the number of image elements. The number of neurons in the hidden layer is usually decided by experiments. Generally, a range of different numbers is tried in the

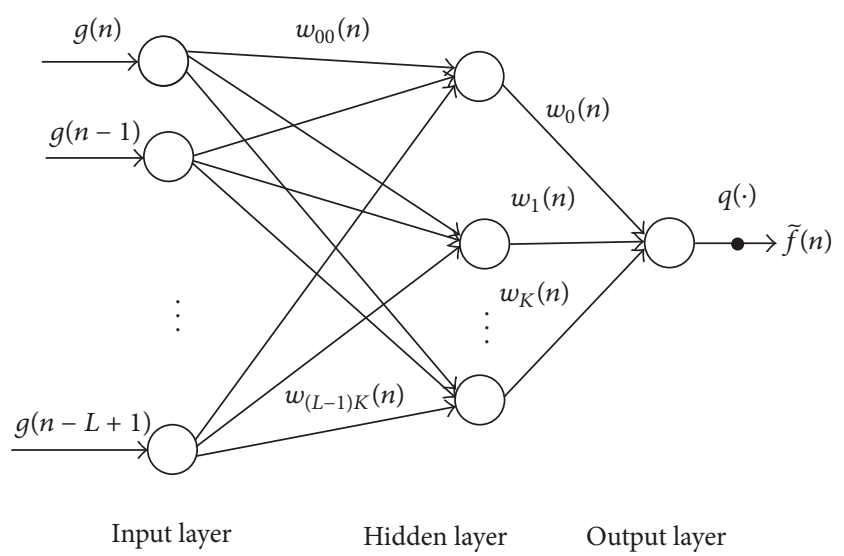

Figure 2: Three-layer feedforward neural network.

hidden layer, and the number that achieves the best training results is selected. The number of neurons in the output layer is one to produce the estimation of the image. The algorithm works by iteratively altering the connection weight values for neurons based on the cost function. The actual modification of weights is carried out using a gradient descent algorithm, where the weights are modified after any iteration.

\section{Feedforward Neural Network Image Blind Equalization Algorithm}

In this section, the feedforward neural network image blind equalization algorithm applied to image restoration is shown in Figure 3.

In Figure 3, we denote 1D sequence transformed from the degraded image via zigzag coding by $g(n) . \tilde{f}(n), \widehat{f}(n)$, and $\widehat{f}_{i j}$ are the output of neural network blind equalizer, the output of the judgment, and the estimator of the image, respectively.

Generally speaking, medical gray CT images are independently distributed source sequences [26]. Vural and Sethares [27] proposed a new method based on nonlinear adaptive filtering for blind deconvolution of noisy blurred images and essentially a $2 \mathrm{D}$ extension of the CMA. The method can either be used to initialize an adaptive blind deconvolution method or be used by itself. Conversely, a 1-D CMA cost function was utilized for the image restoration. The cost function of the observed image takes the form of

$$
J(n)=E\left[\left(|\tilde{f}(n)|^{2}-R_{2}\right)^{2}\right],
$$

where $R_{2}$ is a constant scalar depending on medical CT image.

The proposed algorithm in this paper is implemented by iteratively altering the weights of neural network based on the CMA cost function. The actual modification of weights is carried out by using a gradient descent minimization algorithm, and the modified weights are present in the network after any iteration.

In the wide application of neural network, the choice of transfer function has direct influence on the performance 


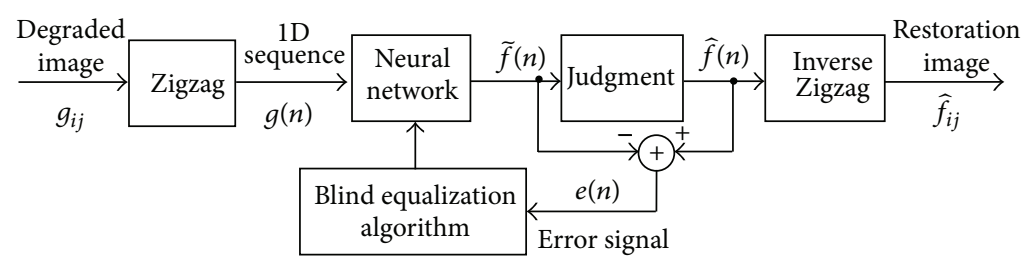

FIGURE 3: Block diagram that describes the flow of the proposed algorithm.

of neural network. There are several most commonly used transfer functions, for instance, the threshold function, the sigmoid function, the hyperbolic tangent function, and so on. In order to improve identification of neural network conveniently applied in the image restoration, the bipolar sigmoid activation function with smooth, gradual, and monotone properties, being conducive to the effective discriminant of the input signal sequence, is selected and utilized in the neural network. The form of transfer function is given by

$$
q(x)=x+\alpha \frac{e^{x}-e^{-x}}{e^{x}+e^{-x}}
$$

where $\alpha$ represents the scale factor that controls classification effect of neural network, and the value of $\alpha$ is usually decided by experiments.

The derivative of $J(n)$ with respect to neural network parameters is needed to implement the gradient descent minimization. The derivative of $J(n)$ with respect to $\mathbf{W}(n)$ can be evaluated, which is given by

$$
\begin{aligned}
\mathbf{W}(n+1) & =\mathbf{W}(n)-\mu \frac{\partial J(n)}{\partial \mathbf{W}(n)} \\
& =\mathbf{W}(n)-2 \mu\left[\tilde{f}^{2}(n)-R_{2}\right] \tilde{f}(n) \frac{\partial \tilde{f}(n)}{\partial \mathbf{W}(n)} .
\end{aligned}
$$

A three-layer feedforward neural network contains different connection weights so that we can obtain different weight iterative formulas.

We denote the weight between the output layer and hidden layer by $w_{k}(n)$; hence, $w_{k}(n)$ is updated according to

$$
\begin{aligned}
w_{k}(n+1)= & w_{k}(n)-2 \mu_{k}\left[\tilde{f}^{2}(n)-R_{2}\right] \tilde{f}(n) q^{\prime}[v(n)] I_{k}(n) \\
= & w_{k}(n)-2 \mu_{k}\left[\tilde{f}^{2}(n)-R_{2}\right] \tilde{f}(n) \\
& \times\left(1+\frac{4 \alpha}{\left(e^{v(n)}+e^{-v(n)}\right)^{2}}\right) I_{k}(n),
\end{aligned}
$$

where $\mu_{k}$ is a scalar parameter.

$w_{l k}(n)$ describes the weight between the input layer and the hidden layer, and then the updating equation is given by

$$
\begin{aligned}
\frac{\partial \tilde{f}(n)}{\partial w_{l k}(n)} & =f^{\prime}[v(n)] \frac{\partial v(n)}{\partial w_{l k}(n)} \\
& =w_{k}(n) q^{\prime}[v(n)] q^{\prime}\left[u_{k}(n)\right] g(n-k)
\end{aligned}
$$

$$
\begin{aligned}
= & w_{k}(n)\left(1+\frac{4 \alpha}{\left(e^{v(n)}+e^{-v(n)}\right)^{2}}\right) \\
& \times\left(1+\frac{4 \alpha}{\left(e^{u_{k}(n)}+e^{-u_{k}(n)}\right)^{2}}\right) g(n-k),
\end{aligned}
$$

where $\mu_{l k}$ represents a scalar factor that controls the variation of the weight.

Equations (7) and (8) constitute the proposed algorithm for blind deconvolution of noisy blurred images. Any iteration of the algorithm corresponds to processing a pixel in the blurred image. When convergence occurs, $\tilde{f}(n)$ provides an estimate of image sequence.

\section{Performance Analysis}

In order to prove the performance of convergence, we define an error function that is illustrated as

$$
\operatorname{erf}(\mathbf{W}(n))=\frac{1}{2} \sum_{n=1}^{N}(\tilde{f}(n)-\hat{f}(n))^{2}
$$

Given some hypothesis conditions,

(A1) $|p(x)|,\left|p^{\prime}(x)\right|$, and $\left|p^{\prime \prime}(x)\right|$ possess boundary continuity for $x \in \mathfrak{R}$;

(A2) there exists a bounded set $\mathbf{D} \subset \mathfrak{R}$, and it makes all the weights of the network meet $\{\mathbf{W}(n)\}_{n=1}^{\infty} \subset \mathbf{D}$;

(A3) the set $\mathbf{D}_{0}=\{\mathbf{W}(n) \in \mathbf{D}: \partial \operatorname{erf}(\mathbf{W}(n)) / \partial \mathbf{W}(n)=0\}$ only contains a finite number of points.

Theorem 1. On the premise of fulfilling learning rate requirements, $\mathbf{W}(n+1)$ iteratively obtained by the gradient descent algorithm and starting from any initial weight can satisfy the following:

(1) $\operatorname{erf}(\mathbf{W}(n+1)) \leq \operatorname{erf}(\mathbf{W}(n))$,

(2) $\lim _{n \rightarrow \infty}\|\partial \operatorname{erf}(\mathbf{W}(n)) / \partial \mathbf{W}(n)\|=0$,

(3) considering network weight matrix, in the case of assumptions (A3) stated, there must be a point $\mathbf{W}^{*} \in$ $\mathbf{D}_{0}$, and $\lim _{n \rightarrow \infty} \mathbf{W}(n)=\mathbf{W}^{*}$.

Here, conclusion (1) ensures monotone of error function when the neural network is utilized to optimize the cost function. Conclusion (2) reflects the convergence property of error function. In other words, the network weights start 
from arbitrary point of the space, and the norm of gradient of error function will eventually converge to 0 . Conclusion (3) indicated that, in the case of existing limited extreme points of the error surface, the weights of the network will eventually converge to the global optimal solution.

Proof. For the sake of certified simplicity, we denote

$$
\begin{gathered}
\varphi_{q, 1}^{n}=\sum_{k=1}^{K}\left\|\sum_{n=1}^{N} \Delta \mathbf{W}(n)\right\|^{q}, \\
\varphi_{q, 1}^{n}=\sum_{k=1}^{K} \sum_{n=1}^{N}\|\Delta \mathbf{W}(n)\|^{q} .
\end{gathered}
$$

Whether assumptions (A1) and (A2) are established, there must exist a constant $\gamma$ satisfying (11) and (12) [28]:

$$
\begin{gathered}
\frac{1}{\mu} \varphi_{q, 1}^{n} \geq \gamma \varphi_{2,2}^{n}, \\
\operatorname{erf}(\mathbf{W}(n+1)) \leq \operatorname{erf}(\mathbf{W}(n))-\frac{1}{\mu} \varphi_{q, 1}^{n}+\gamma \varphi_{2,2}^{n}, \\
\varsigma^{n}=\frac{1}{\mu} \varphi_{q, 1}^{n}-\gamma \varphi_{2,2}^{n} .
\end{gathered}
$$

(11) and (12) ensure that conclusion (1) was deduced.

From (12), we have drawn

$$
0 \leq \operatorname{erf}(\mathbf{W}(n+1)) \leq \operatorname{erf}(\mathbf{W}(n))-\varsigma^{n} \leq \operatorname{erf}(\mathbf{W}(N))-\sum_{n=1}^{N} \varsigma^{n},
$$

$\sum_{n=1}^{N} \varsigma^{n} \leq \operatorname{erf}(\mathbf{W}(N))$, when $N \rightarrow \infty$, then

$$
\sum_{n=1}^{\infty} \varsigma^{n} \leq \operatorname{erf}(\mathbf{W}(N))<\infty
$$

Whether assumptions (A1) and (A2) were established, there must be a constant $c_{1}$ satisfying (16) and a constant $c_{2}$ satisfying (17):

$$
\begin{gathered}
\sum_{n=1}^{\infty} \frac{1}{n}\left\|\frac{\partial \operatorname{erf}(\mathbf{W}(n))}{\partial \mathbf{W}(n)}\right\|^{2} \leq c_{1} \sum_{n=1}^{\infty}\left(\frac{1}{\mu} \varphi_{2,1}^{n}\right)<\infty, \\
\sum_{k=1}^{K} \sum_{n=1}^{N}\|\Delta \mathbf{W}(n)\| \leq c_{2} .
\end{gathered}
$$

The mean value theorem was, respectively, adopted for $p(x)$ and $p^{\prime}(x)$, and there exists a constant $c_{3}$ satisfying the following equation:

$$
\left\|\frac{\partial \operatorname{erf}(\mathbf{W}(n+1))}{\partial \mathbf{W}}\right\|-\left\|\frac{\partial \operatorname{erf}(\mathbf{W}(n))}{\partial \mathbf{W}}\right\| \mid \leq c_{3} \sum_{k=1}^{K}\left\|\mathbf{d}_{k}\right\|,
$$

where $\mathbf{d}_{k}=\mathbf{W}_{k}(n+1)-\mathbf{W}_{k}(n)$.
From (17) and (18), we can obtain

$$
\lim _{n \rightarrow \infty}\left\|\frac{\partial \operatorname{erf}\left(\mathbf{W}_{k}(n)\right)}{\partial \mathbf{W}_{k}(n)}\right\|=0 .
$$

Resembling (18), there is a constant $c_{4}$ satisfying the following equation:

$$
\left\|\frac{\partial \operatorname{erf}\left(\mathbf{W}_{k+l}(n+1)\right)}{\partial \mathbf{W}}-\frac{\partial \operatorname{erf}\left(\mathbf{W}_{k}(n)\right)}{\partial \mathbf{W}}\right\| \leq \frac{c_{4}}{l} .
$$

Using (16) and (17), we have

$$
\lim _{n \rightarrow \infty}\left\|\frac{\partial \operatorname{erf}\left(\mathbf{W}_{k+l}(n)\right)}{\partial \mathbf{W}}\right\|=0
$$

Therefore, conclusion (2) was deduced from the premises. From (18), we have drawn

$\lim _{n \rightarrow \infty}\left\|\mathbf{W}_{k}(n+1)-\mathbf{W}_{k}(n)\right\|=0, \quad k=1,2, \ldots, K$.

As mentioned perviously

$\|\mathbf{W}(n+1)-\mathbf{W}(n)\| \leq \sum_{k=1}^{K}\left\|\mathbf{W}_{k}(n+1)-\mathbf{W}_{k}(n)\right\|$.

Thus,

$$
\lim _{n \rightarrow \infty}\|\mathbf{W}(n+1)-\mathbf{W}(n)\|=0 .
$$

To prove this, a lemma was introduced [28].

Lemma 2. $E: \Re^{M N+M+1} \rightarrow \mathfrak{R}$ is a uniformly differentiable function with a set $D \subset \mathfrak{R}^{M N+M+1}$. The mathematical set $D_{0}=$ $\{\mathbf{W} \in D \mid \partial E(\mathbf{W}) / \partial \mathbf{W}=0\}$ only contains limited points. The sequence of $\left\{\mathbf{W}^{m}\right\}_{m=1}^{\infty} \subset D$ satisfies the following conditions:

$$
\begin{gathered}
\lim _{m \rightarrow \infty}\left\|\mathbf{W}^{m+1}-\mathbf{W}^{m}\right\|=0, \\
\lim _{m \rightarrow \infty}\left\|\frac{\partial E\left(\mathbf{W}^{m}\right)}{\partial \mathbf{W}}\right\|=0 .
\end{gathered}
$$

Then, there exists a point $\mathbf{W}^{*} \subset D_{0}$ if satisfying the following condition:

$$
\lim _{m \rightarrow \infty} \mathbf{W}^{m}=\mathbf{W}^{*} .
$$

Because $\operatorname{erf}(\mathbf{W}(n))$ is a uniformly differentiable function, from (24) and the lemma, we can see that there exists $\mathbf{W}^{*} \in \mathbf{D}_{0}$ that satisfies

$$
\lim _{n \rightarrow \infty} \mathbf{W}(n)=\mathbf{W}^{*} .
$$

Hence, it is not difficult to conclude the above.

\section{Experimental Result}

We illustrate the performance of the proposed method on handling noisy degraded medical CT images in the first 


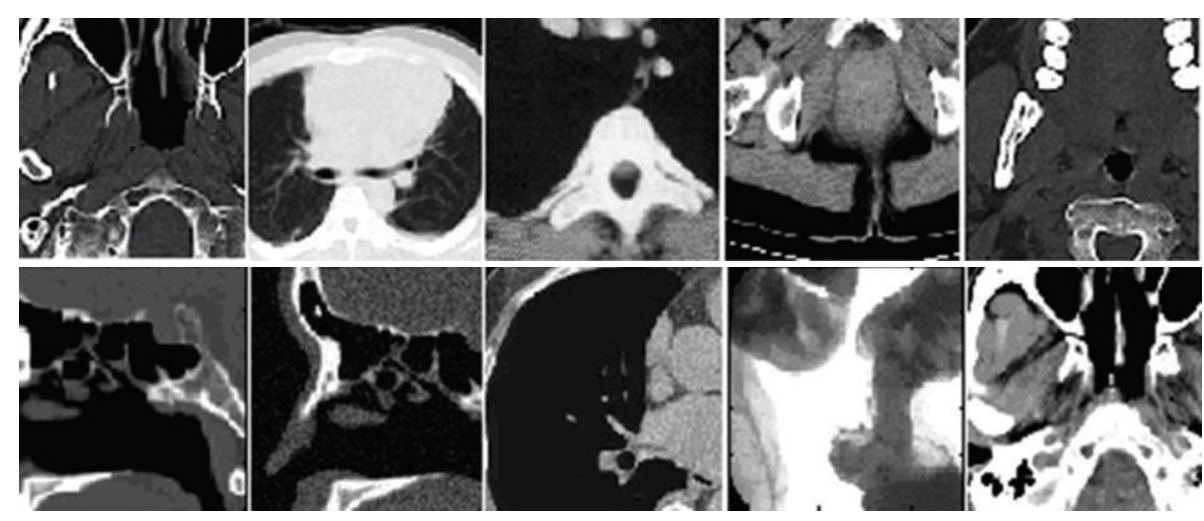

FIGURE 4: A set of images used for synthetic experiments.

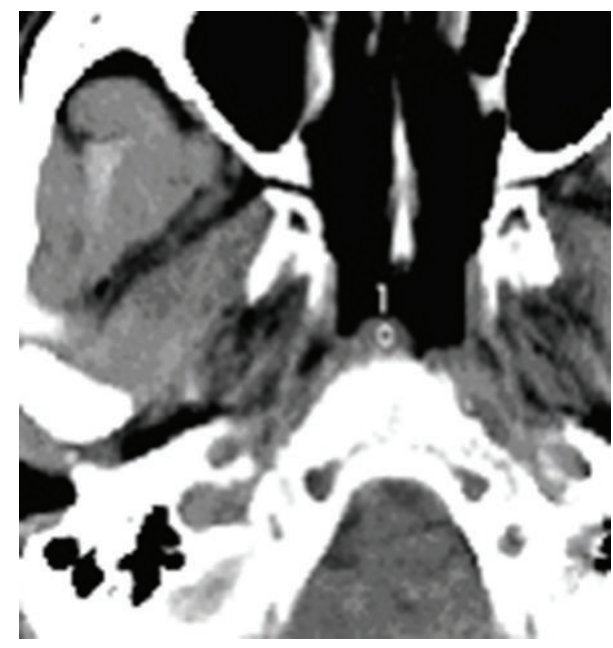

(a)

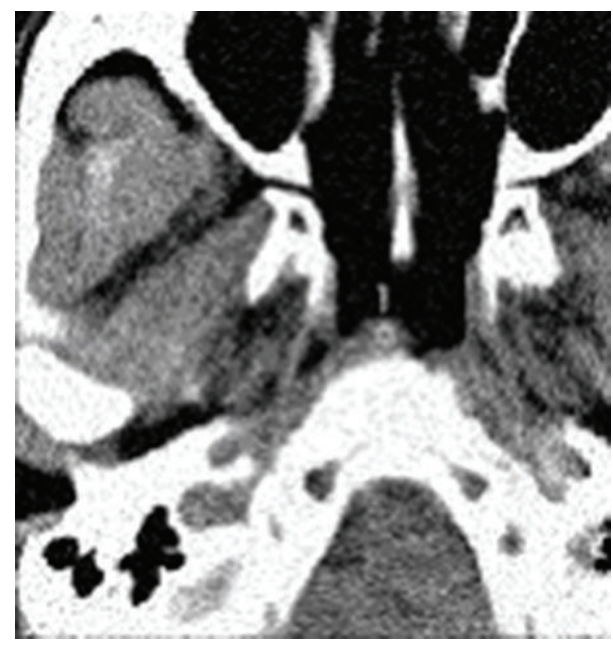

(b)

FIgURE 5: (a) Original image. (b) Noise blurred image.

experiment. Ten images used in this experiment are shown in Figure 4. Each blurred image is generated by applying a Gaussian blurring kernel with standard deviation 0.001 to the original image first and then followed by Gaussian i.i.d. noise at a blurred signal-to-noise ratio (BSNR) [29] of $20 \mathrm{~dB}$. An 11-5-1 network is adopted for the problem that we effectively overcome the effect of point spread function and restore the image with functions in (5) as transfer function. We set all of the step size to 0.0001 in the first test. The network is trained with random initial weights selected from the interval [ $\left[\begin{array}{ll}0 & 1\end{array}\right]$, and the training is terminated when the RMSE per epoch reaches 0.01 . It is usually necessary to complete the training process for all images, respectively, and all of the training processes need about 75 iterations, respectively, in this experiment. The ISNR values range from $0.39 \mathrm{~dB}$ to $0.97 \mathrm{~dB}$. On a more theoretical level, the choice standard of CT images that may have an influence on the performance of the proposed method is another important topic for further discussion.

The following experiments display the comparison of performance between our proposed method and other methods. The sectional CT image of nasal pharynx chronic inflammation is utilized to illustrate the effectiveness of the proposed algorithm. In a second experiment, the original images of size $256 \times 256$ shown in Figure 5(a) were degraded by a $25 \times 25$ Gaussian blur with a standard deviation of 0.002 , followed by a $20 \mathrm{~dB}$ additive noise to form the noisy blurred images shown in Figure 5(b). In this experiment, we chose to use conjugate gradient to optimize an 11-5-1 feedforward neural network adopted in the simulation. The value of the step size between the input layer and hidden layer is $\mu_{1}=5 \times$ $10^{-7}$, and the value between the output layer and hidden layer is $\mu_{2}=1 \times 10^{-7}$. The rest of network training is similar to the first experiment. Note that the value of $R_{2}$ depends on the grey level of image and has been manually fixed, $R_{2}=39320.0$ [27].

We compare the results of the proposed algorithm with iterative blind deconvolution algorithm, dispersion minimization algorithm, and reduction dimension constant module algorithm. The number of iterations of IBD is 100 and the restored image is shown in Figure 6(a). The restored images of dispersion minimization algorithm and reduction dimension constant module algorithm are depicted in Figures 6(b) and 6(c), respectively. Figure 6(d) shows the result of the proposed algorithm. The restored image proposed in this paper is 


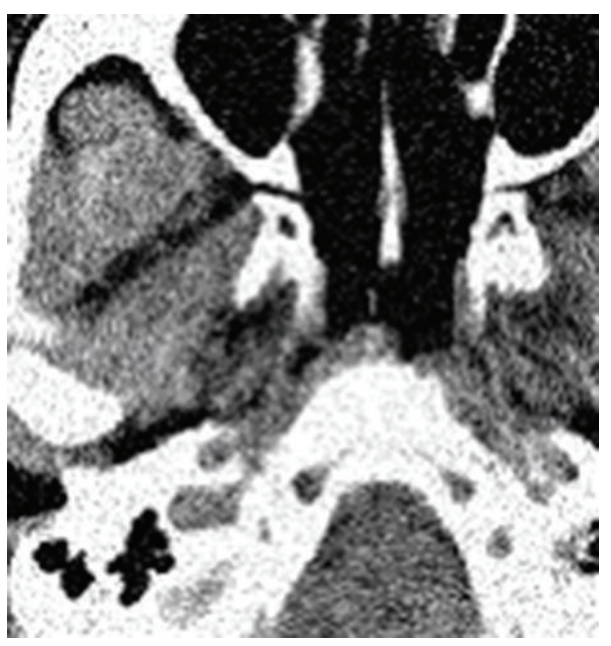

(a)

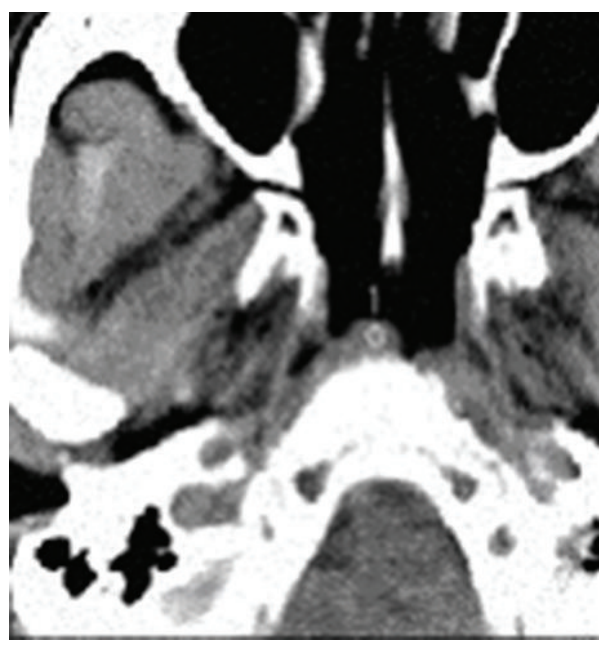

(c)

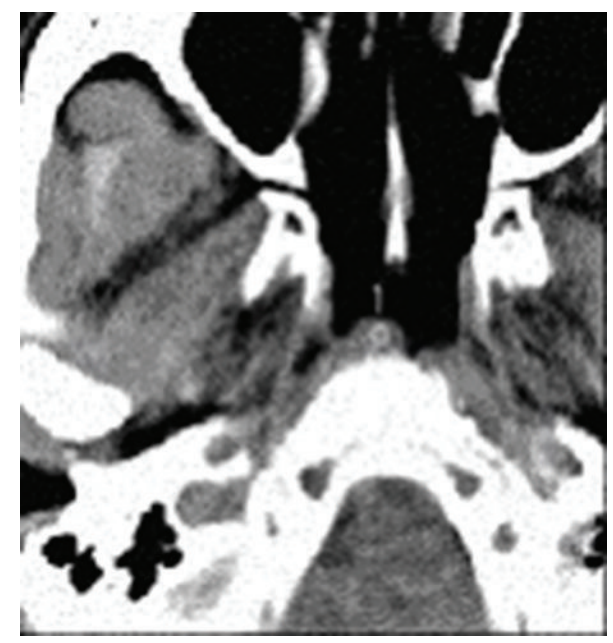

(b)

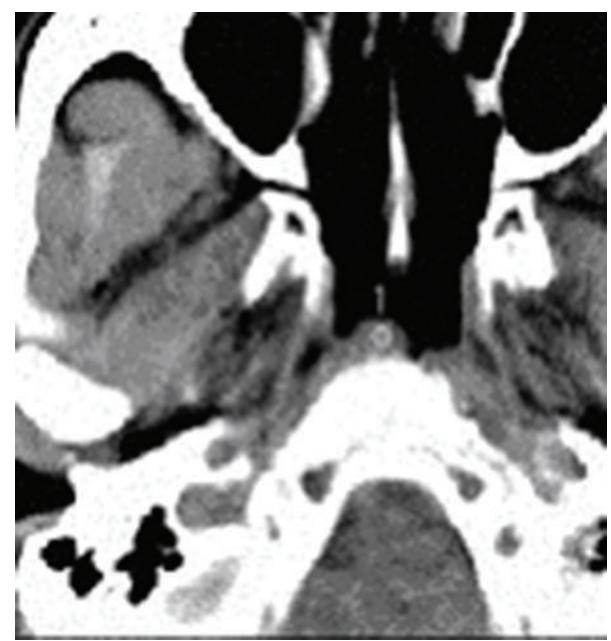

(d)

FIGURE 6: Restored image. (a) IBD algorithm. (b) Dispersion minimization algorithm. (c). Reduction dimension constant module algorithm. (d) The proposed algorithm.

TABLE 1: The comparison of performance.

\begin{tabular}{lcccc}
\hline Method & The proposed algorithm & $\begin{array}{c}\text { Dispersion minimization } \\
\text { algorithm }\end{array}$ & $\begin{array}{c}\text { Reduction dimension constant } \\
\text { module algorithm }\end{array}$ & $\begin{array}{c}\text { Iterative blind } \\
\text { deconvolution algorithm }\end{array}$ \\
\hline ISNR & 1.0622 & 0.9736 & 0.9986 & 0.7488 \\
\hline
\end{tabular}

TABLE 2: The comparison of performance.

\begin{tabular}{lcccc}
\hline Noise BSNR & The proposed algorithm & $\begin{array}{c}\text { Dispersion minimization } \\
\text { algorithm }\end{array}$ & $\begin{array}{c}\text { Reduction dimension constant } \\
\text { module algorithm }\end{array}$ & $\begin{array}{c}\text { Iterative blind } \\
\text { deconvolution algorithm }\end{array}$ \\
\hline $20 \mathrm{~dB}$ & 0.9064 & 0.8362 & 0.8485 & 0.8182 \\
$27 \mathrm{~dB}$ & 0.9065 & 0.8762 & 0.8614 & 0.8082 \\
\hline
\end{tabular}

closer to the original image than the other ones. Overall, these results show that the proposed method has better performance on preserving the image characteristics in the restoration process.
To quantitatively evaluate the restored images, the metric of the improvement signal-to-noise ratio (ISNR) [30] is employed. The performance comparison between various methods is tabulated in Table 1. Comparing with the original image 


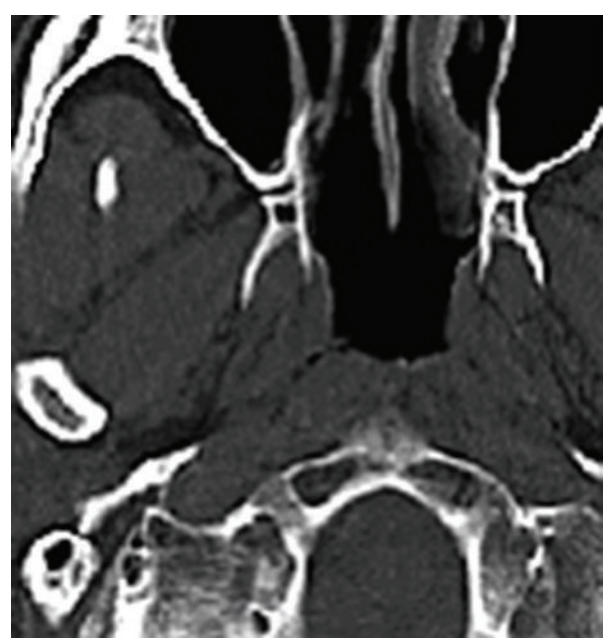

Figure 7: The original CT image.

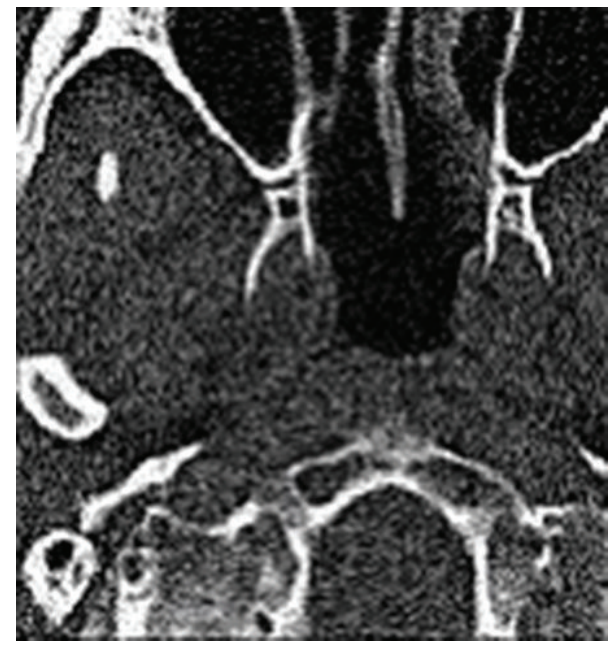

(a)

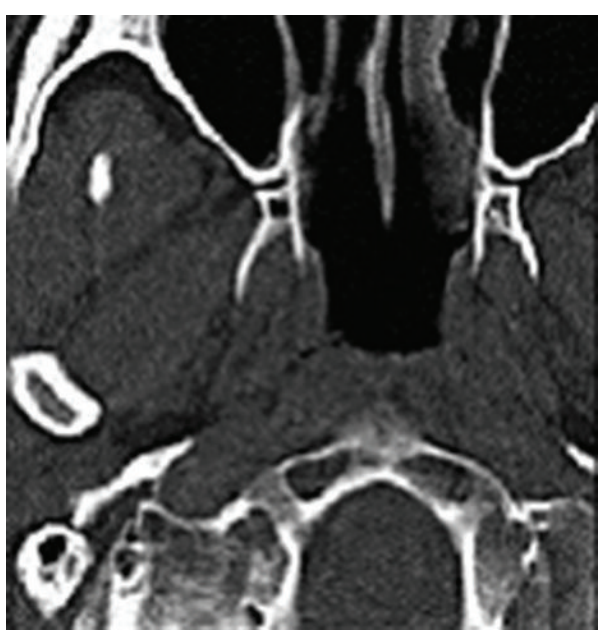

(c)

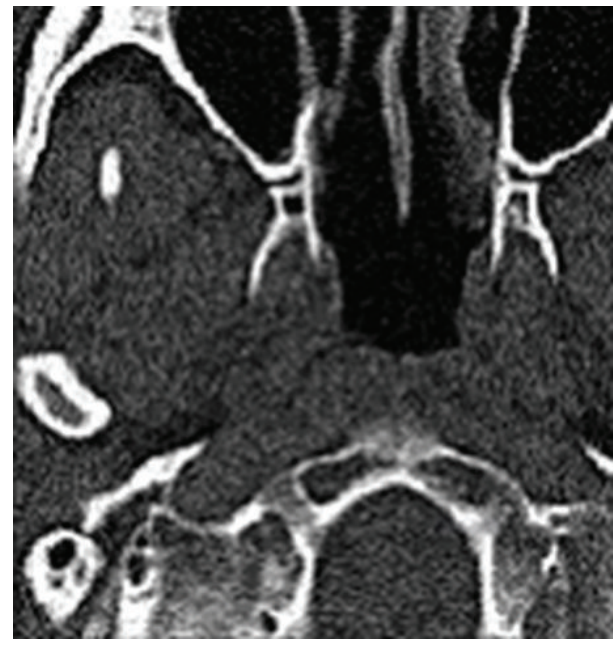

(b)

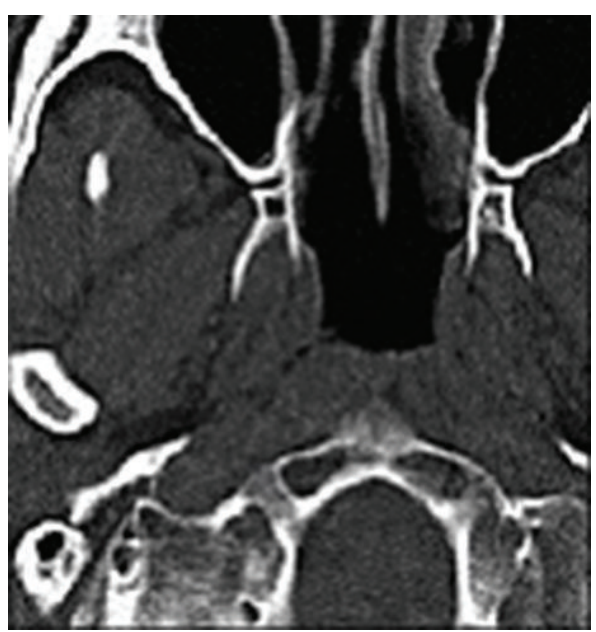

(d)

FIgURE 8: (a) Noise blurred image at $20 \mathrm{~dB}$ BSNR. (b) Noise blurred image at $27 \mathrm{~dB}$ BSNR. (c) Restored image by the proposed algorithm for noise blurred image at $20 \mathrm{~dB}$ BSNR. (d) Restored image by the proposed algorithm for noise blurred image at $27 \mathrm{~dB}$ BSNR. 
in Figure 5(a), it is clear that the proposed algorithm can provide restored images with very high visual quality. ISNR values of various methods, whose ideal values are $+\infty$, are presented in row 2 of Table 1 . The larger the value of ISNR is, the higher the image quality is. It is clear shown in Table 1 that the proposed method performs better than the other approaches.

In the third experiment, the original image shown in Figure 7 was degraded with $10 \times 10$ Gaussian blur with a standard deviation of 0.05 followed by different additive noises to form the noisy blurred image in Figures 8(a) and 8(b). In this experiment, we chose to utilize the same network structure as the first one. Here, the step size factor between the input layer and hidden layer is $\mu_{1}=1 \times 10^{-9}$, and the one between the output layer and hidden layer is $\mu_{2}=2 \times 10^{-8}$. The rest of simulation conditions and network training are similar to the second experiment.

The performance of the proposed method compared with the performance of other three methods including dispersion minimization algorithm, reduction dimension constant module algorithm, and iterative blind deconvolution algorithm is presented in Table 2. It is also clear seen in Table 2 that the proposed method performs better than some methods having been put out.

The proposed algorithm makes use of Zigzag coding to transform the degraded image into a signal sequence, and then the neural network blind equalization algorithm completes the elimination of point spread function. Even though by contrast the proposed algorithm is computationally complex, we can see that the method yielded, in almost all cases, a significant improvement in image quality. An illustrative example shows that the method has its practicability and maneuverability. Moreover, the improved hardware remedies repair the defect of the proposed algorithm on high computational complexity.

\section{Conclusions}

In this work, the neural network blind equalization for image restoration has been extended to a $2 \mathrm{D}$ case. Using blind equalization to overcome the effect of PSF is equivalent to eliminating ISI. We propose to utilize Zigzag coding to transform degraded image into 1-dimensional signal sequence, construct constant modulus cost function applied to grayscale CT image, and optimize the cost function to address blind image restoration. Experimental results have showed that the proposed method performs quite well in terms of both visual inspection and quantitative evaluation such as improvement peak signal to noise ratio. In addition, several issues will be further explored. We will study the sensitivity of the presented method towards the selection of parameters. We will also use real-life images to further evaluate the performance of the proposed method.

\section{Acknowledgments}

The authors thank the editor and the anonymous reviewers for their helpful comments and valuable suggestions during the revision of this paper. This work was supported in part by Tianjin College Science and Technology Development Foundation of China under Grant (20110709) and by the Shanxi Natural Science Foundation under Grant (2011011015-3).

\section{References}

[1] Y. V. Zhulina, "Multiframe blind deconvolution of heavily blurred astronomical images," Applied Optics, vol. 45, no. 28, pp. 7342-7352, 2006.

[2] A. Jalobeanu, L. Blanc-Féraud, and J. Zerubia, "Hyperparameter estimation for satellite image restoration using a MCMC maximum-likelihood method," Pattern Recognition, vol. 35, no. 2, pp. 341-352, 2002.

[3] H. Shen, L. Du, L. Zhang, and W. Gong, "A blind restoration method for remote sensing images," IEEE Geoscience and Remote Sensing Letters, vol. 9, no. 6, pp. 1137-1141, 2012.

[4] R. Fergus, B. Singh, A. Hertzmann, S. T. Roweis, and W. T. Freeman, "Removing camera shake from a single photograph," $A C M$ Transactions on Graphics (TOG), vol. 25, pp. 787-794, 2006.

[5] O. Michailovich and A. Tannenbaum, "Blind deconvolution of medical ultrasound images: a parametric inverse filtering approach," IEEE Transactions on Image Processing, vol. 16, no. 12, pp. 3005-3019, 2007.

[6] R. Jiř́k and T. Taxt, "Two-dimensional blind Bayesian deconvolution of medical ultrasound images," IEEE Transactions on Ultrasonics, Ferroelectrics, and Frequency Control, vol. 55, no. 10, pp. 2140-2153, 2008.

[7] W. Souidene, K. Abed-Meraim, and A. Beghdadi, "A new look to multichannel blind image deconvolution," IEEE Transactions on Image Processing, vol. 18, no. 7, pp. 1487-1500, 2009.

[8] D. Li, R. M. Mersereau, and S. Simske, "Blind image deconvolution through support vector regression," IEEE Transactions on Neural Networks, vol. 18, no. 3, pp. 931-935, 2007.

[9] S. J. Reeves and R. M. Mersereau, "Blur identification by the method of generalized cross-validation," IEEE Transactions on Image Processing, vol. 1, no. 3, pp. 301-311, 1992.

[10] R. G. Lane and R. H. T. Bates, "Automatic multidimensional deeonvolution," Journal of the Optical Society of America A, vol. 4, no. 1, pp. 180-188, 1987.

[11] D. G. Tzikas, A. C. Likas, and N. P. Galatsanos, "Variational bayesian sparse kernel-based blind image deconvolution with Student's-t priors," IEEE Transactions on Image Processing, vol. 18, no. 4, pp. 753-764, 2009.

[12] K.-H. Yap and L. Guan, "A computational reinforced learning scheme to blind image deconvolution," IEEE Transactions on Evolutionary Computation, vol. 6, no. 1, pp. 2-15, 2002.

[13] M. Jiang, G. Wang, M. W. Skinner, J. T. Rubinstein, and M. W. Vannier, "Blind deblurring of spiral CT images," IEEE Transactions on Medical Imaging, vol. 22, no. 7, pp. 837-845, 2003.

[14] E. Y. Lam, "Blind bi-level image restoration with iterated quadratic programming," IEEE Transactions on Circuits and Systems II, vol. 54, no. 1, pp. 52-56, 2007.

[15] D. Kundur and D. Hatzinakos, "On the use of Lyapunov criteria to analyze the convergence of blind deconvolution algorithms," IEEE Transactions on Signal Processing, vol. 46, no. 11, pp. 29182925, 1998. 
[16] C. A. Ong and J. A. Chambers, "An enhanced NAS-RIF algorithm for blind image deconvolution," IEEE Transactions on Image Processing, vol. 8, no. 7, pp. 988-992, 1999.

[17] B. C. McCallum, "Blind deconvolution by simulated annealing," Optics Communications, vol. 75, no. 2, pp. 101-105, 1990.

[18] J. Wang, K. Lu, Q. Wang, and J. Jia, "Kernel optimization for blind motion deblurring with image edge prior," Mathematical Problems in Engineering, vol. 2012, Article ID 639824, 10 pages, 2012.

[19] T. A. Cheema, I. M. Qureshi, A. Jalil, and A. Naveed, "Artificial neural networks for blur identification and restoration of nonlinearly degraded images," International Journal of Neural Systems, vol. 11, no. 5, pp. 455-461, 2001.

[20] B. Karlik, "Medical image compression by using Vector Quantization Neural Network (VQNN)," Neural Network World, vol. 16, no. 4, pp. 341-348, 2006.

[21] I. Aizenberg, D. V. Paliy, J. M. Zurada, and J. T. Astola, "Blur identification by multilayer neural network based on multivalued neurons," IEEE Transactions on Neural Networks, vol. 19, no. 5, pp. 883-898, 2008.

[22] B. Zineddin, Z. Wang, and X. Liu, "Cellular neural networks, the Navier-Stokes equation, and microarray image reconstruction," IEEE Transactions on Image Processing, vol. 20, no. 11, pp. 32963301, 2011.

[23] Y. D. Wu, Y. Sun, H. Y. Zhang, and S. X. Sun, "Variational PDE based image restoration using neural network," IET Image Processing, vol. 1, no. 1, pp. 85-93, 2007.

[24] L. Zhang, H. Cheng, and Y. Bai, "A neural network blind equalization algorithm based on variable momentum factor," in Proceedings of the IET Conference on Wireless, Mobile and Sensor Networks (CCWMSN '07), pp. 229-232, December 2007.

[25] T. Tunc, "A new hybrid method logistic regression and feedforward neural network for lung cancer data," Mathematical Problems in Engineering, vol. 2012, Article ID 241690, 10 pages, 2012.

[26] D. Pradeepa Samarasinghe and A. Rodney, "Kurtosis CMA deconvolution for blind image restoration," in Proceedings of the 4th International Conference on Information and Automation for Sustainablity, pp. 271-276, Colombo, SriLanka, 2008.

[27] C. Vural and W. A. Sethares, "Blind image deconvolution via dispersion minimization," Digital Signal Processing, vol. 16, no. 2, pp. 137-148, 2006.

[28] W. Wu, G. Feng, Z. Li, and Y. Xu, "Deterministic convergence of an online gradient method for BP neural networks," IEEE Transactions on Neural Networks, vol. 16, no. 3, pp. 533-540, 2005.

[29] M. S. C. Almeida and L. B. Almeida, "Blind and semi-blind deblurring of natural images," IEEE Transactions on Image Processing, vol. 19, no. 1, pp. 36-52, 2010.

[30] A.-K. Seghouane, "A Kullback-Leibler divergence approach to blind image restoration," IEEE Transactions on Image Processing, vol. 20, no. 7, pp. 2078-2083, 2011. 


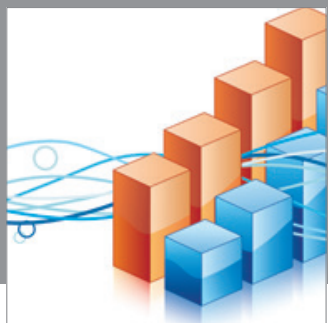

Advances in

Operations Research

mansans

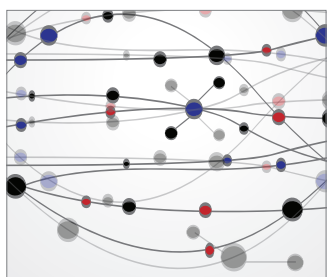

The Scientific World Journal
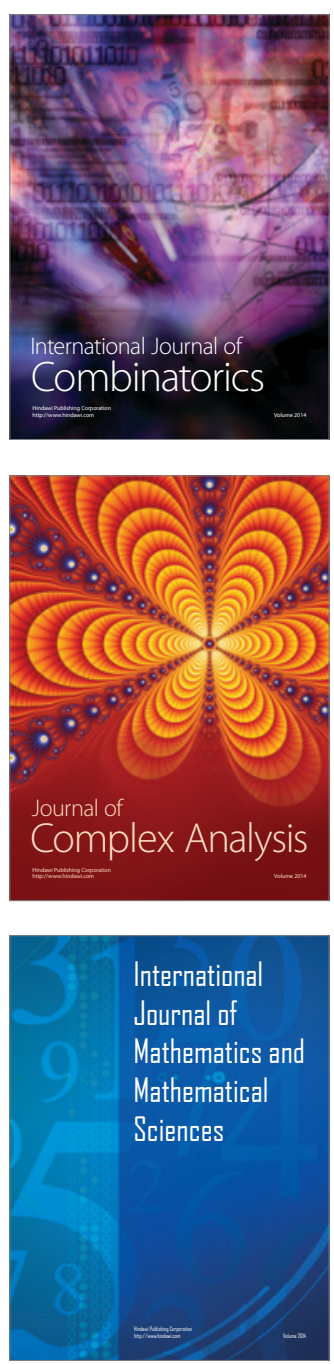
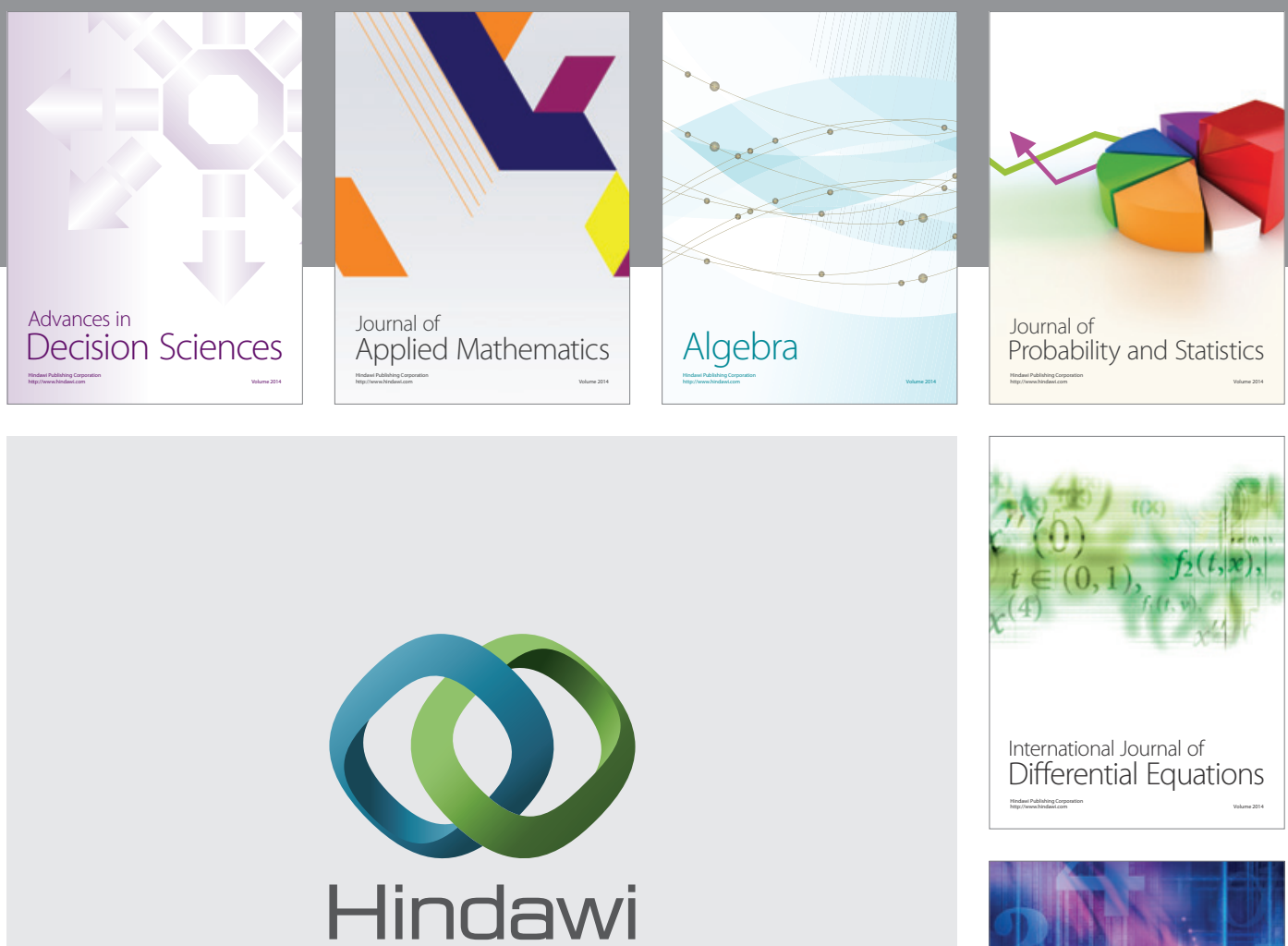

Submit your manuscripts at http://www.hindawi.com
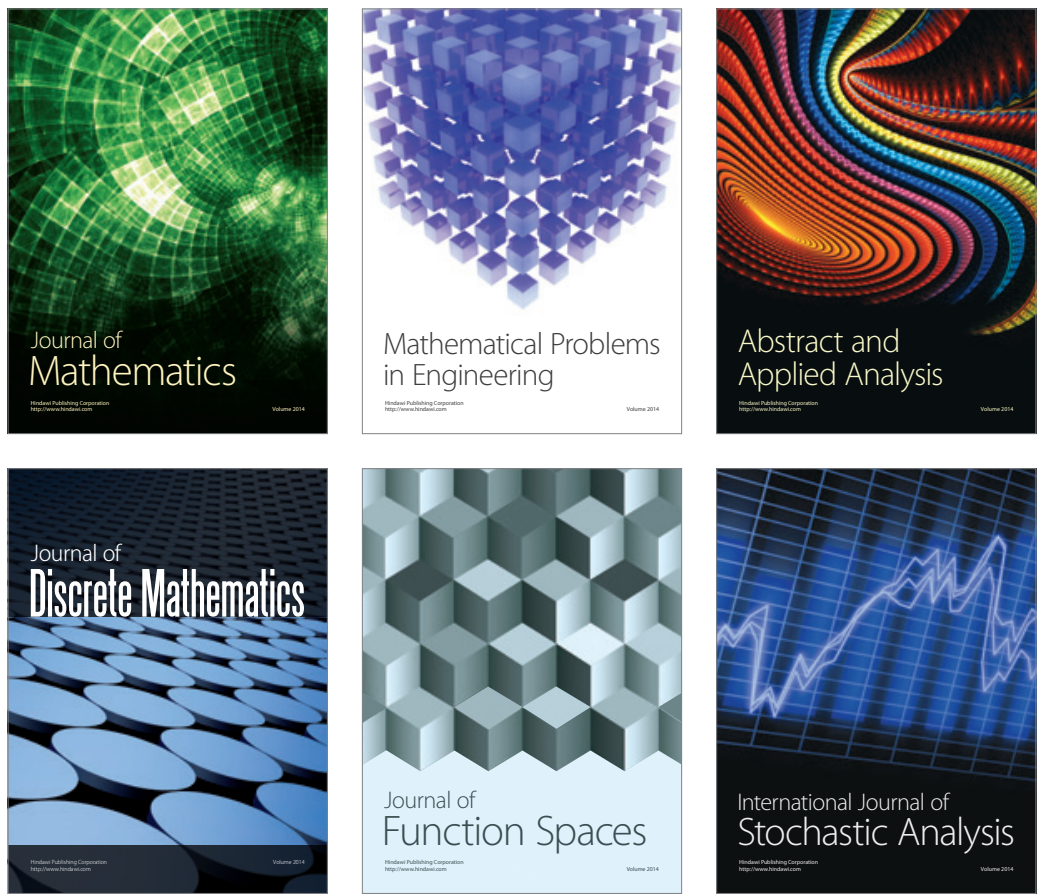

Journal of

Function Spaces

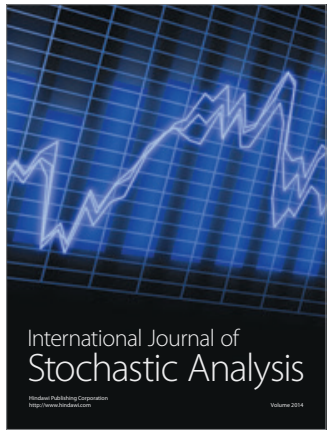

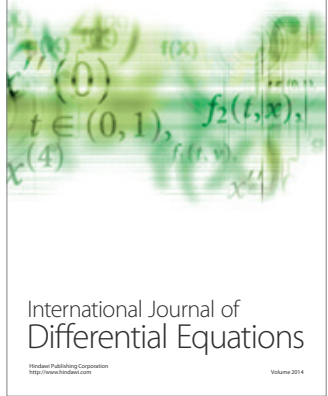
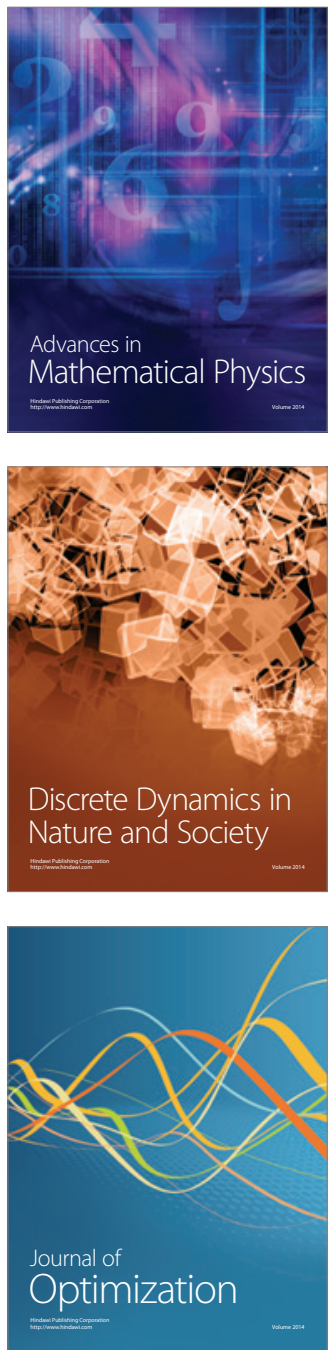